Open Access

\title{
Allergy immunotherapy prescribing trends for grass pollen-induced allergic rhinitis in Germany: a retrospective cohort analysis
}

Amanda L. McDonell ${ }^{1 *}$, Ulrich Wahn², Dirk Demuth', Catrina Richards ${ }^{1}$, Charlie Hawes ${ }^{3}$, Jakob Nørgaard Andreasen ${ }^{4}$ and Felicia Allen-Ramey ${ }^{5}$

\begin{abstract}
Background: Allergy immunotherapy is an effective treatment for patients with allergic rhinitis whose symptoms are unresolved with pharmacotherapy. Allergy immunotherapy for grass pollen-induced allergic rhinitis is available in three modalities: subcutaneous immunotherapy and sublingual immunotherapy as a tablet or drop. This study aimed to understand trends in allergy immunotherapy prescribing and practice patterns for grass allergies in adult and paediatric patients in Germany.

Methods: A retrospective cohort study was conducted using IMS Disease Analyzer in Germany. Patients with an allergy immunotherapy prescription for grass pollen (Anatomical Therapeutic Chemical [ATC] classification code V01AA02) from September 2005 to December 2012 were included in the study. General Practitioners (GPs), dermatologists, Ear, Nose and Throat (ENT)-specialists, paediatricians and pneumologists were included as the allergy immunotherapy prescribing physicians in the study. Descriptive analyses were conducted on patient characteristics at index and prescribing physician specialty; a test for trend was conducted for timing of initiation of first allergy immunotherapy prescription in each annual prescribing season.

Results: Eighteen thousand eight hundred fifty eligible patients were identified during the study period. The majority of patients received subcutaneous immunotherapy; however, the proportion of patients receiving sublingual immunotherapy tablets increased from $8 \%$ in 2006/2007 to $29 \%$ in 2011/2012 ( $p<0.001)$. Initiation of subcutaneous immunotherapy and Oralair ${ }^{\circledast}$ generally peaked during each prescribing year in two seasons (September-October and January) while GRAZAX ${ }^{\circledR}$ prescriptions peaked in autumn (September-October). ENT-specialists and dermatologists were the largest allergy immunotherapy prescribers in adults, while paediatricians and ENT-specialists were the largest prescribers of allergy immunotherapy in paediatric patients.

Conclusions: Subcutaneous immunotherapy remained the dominant allergy immunotherapy modality for grass pollen-induced allergic rhinitis in Germany for adult and paediatric patients; however, there was a marked increase in proportion of patients receiving sublingual immunotherapy tablets from 2006/2007 to 2011/2012, after their introduction to the market in 2006. ENT-specialists, dermatologists and paediatricians were responsible for the majority of prescribing. The predominance of particular modalities within certain physician specialties likely reflects different treatment goals or needs.
\end{abstract}

Keywords: Allergens, Clinical immunology, Paediatrics, Grass pollen allergy, Rhinitis

\footnotetext{
* Correspondence: AMcDonell@uk.imshealth.com

${ }^{1}$ Real-World Evidence Solutions and Health Economics \& Outcomes Research,

IMS Health, 210 Pentonville Road, London N1 9JY, UK

Full list of author information is available at the end of the article
} 


\section{Background}

Allergic rhinitis (AR) affects $23 \%$ of the population in Western Europe, with approximately half affected by grass pollen-induced rhinitis [1]. Allergy Immunotherapy (AIT) desensitizes the immune system to allergens (including grass pollen) and is a treatment option to alleviate AR symptoms when a patient is not adequately managed with symptomatic medication. The benefits of AIT can include symptom reduction, reduced need for symptom relieving medications, disease modification, improvement in asthma symptoms, and prevention of new sensitizations and allergic asthma [2-13]. AIT is available in three modalities: subcutaneous immunotherapy (SCIT) and sublingual immunotherapy (SLIT) in both a tablet (SLIT-tablet) and drop (SLIT-drop) formulation. SLIT-tablet is a relatively new modality, with two products (GRAZAX ${ }^{\circ}$ : standardized allergen extract of grass pollen from Phleum pratense 75,000 Standardized Quality units Tablet per oral lyophilisate; and Oralair : 5-grass pollen allergen extract tablet, 100 index of reactivity [IR] \& 300 IR) available in European countries since 2006 and 2008, respectively $[14,15]$.

AIT is effective and well tolerated in adults and children, and the Joint Task Force on Practice Parameters recommends that AIT can be considered for patients who have demonstrable evidence of specific immunoglobulin E antibodies to clinically relevant allergens [16]. Although the comparative efficacy of SCIT and SLIT has not been established with direct comparative randomized controlled trials, the clinical efficacy of SLIT (irrespective of allergen) has been suggested to be similar to SCIT based on select open, controlled trials in an evidence review [17]; however, a review of meta-analyses has suggested greater efficacy of SCIT [18], while other studies have found no statistically significant difference between modalities [19].

In practice, choice of modality is likely based on patient and prescriber preferences. SCIT allows more time for patient-physician interaction as it is physicianadministered and can treat multiple allergens at once; however, the treatment regimen is lengthy (generally $3-$ 5 years), the frequency of visits may be inconvenient, and the modality unattractive to patients with needle phobia [20]. Although speculative, given that SLITtablets have not been available in the United States (US) until recently, SCIT may be found to be preferred amongst allergists in the US as these physicians typically formulate extracts themselves; whereas in Europe virtually all AIT is formulated by extract manufacturers [21].

The three AIT modalities (SCIT, SLIT-drops, SLITtablets) have been available for several years in Germany, with treatment initiated by a range of physician specialties, some with sub-specialty in allergology. In the present study we describe the AIT prescribing and practice patterns for paediatric and adult grass pollen-induced AR patients in Germany. The study covers the period preand post-introduction of SLIT tablets, thus enabling the study of the impact of this new modality on prescribing patterns.

\section{Methods \\ Study design and data source}

This was a retrospective cohort study using the IMS Disease Analyzer (DA) database in Germany. DA collects data from Electronic Medical Records of General Practitioners (GP) and office-based specialists and has been shown to be representative of the German population [22]. GPs, dermatologists, Ear, Nose and Throat (ENT)-specialists, paediatricians, and pneumologists were included in this study. Dermatologists were included based on their history as the original allergists in Germany and paediatricians based on the frequency of allergology training in this specialty. Allergology is a sub-specialty in Germany, rather than a distinct specialty (e.g., paediatricians can have sub-specialty in allergology); this is in contrast to some countries where allergology is a distinct specialty (e.g., US).

\section{Patient inclusion criteria}

Patients were included in the study if they received an AIT prescription with an Anatomical Therapeutic Chemical (ATC) classification code for grass pollen (V01AA02) during the study period. Patients were excluded if they had multiple AIT modalities prescribed at index or had a record of an insect allergy (ATC V01AA07), since insect venom AIT regimens differ from that of seasonal allergies. There were no criteria for inclusion or exclusion of patients receiving multi-allergen immunotherapy.

\section{Analyses}

To meet the study objectives, two specific analyses were conducted. The first was a cross-sectional analysis to determine temporal trends of AIT during each "prescribing year" (1 September - 31 August annually) from 2005 to 2012. The prescribing year was designated to begin in September based on clinical input regarding customary approach to AIT initiation in Germany, as prescriptions started in September are intended to alleviate symptoms in the upcoming season (co-author: U. Wahn). The same patient could have been included in analyses across years, but only their first prescription each year was included in annual analyses. Patients were grouped by modality with SLIT-tablets segmented into GRAZAX ${ }^{\bullet}$ and Oralair.

McNemar's test of difference between first and last year of the study period and Chi-squared test for trend over time were used to determine statistical significance of differences in proportion of patients prescribed each 
modality between years. Initial and total AIT prescriptions were compared within each year to observe differences in trend that may have arisen from specification of the prescribing year.

A second analysis was undertaken to examine patient characteristics and physician specialities related to AIT. The first AIT prescription identified for a patient between September 1st 2005 and December 31st 2012 was considered the index prescription. Medical records prior to index were searched for prior AIT use. Information on the physician associated with the index prescription determined physician specialty. The proportion of patients prescribed each modality by physician type and age group was calculated over the entire study period and in the calendar year of 2012 alone representing the most recent year. Descriptive statistics for number of AIT patients treated by each prescribing physician were also calculated.

Patients were characterized at index by age, gender, co-morbidities, history of grass pollen AIT use, and insurance status. Paediatric patients were $<18$ years of age at index. A diagnosis code of a relevant co-morbidity (asthma, sinusitis, atopic dermatitis, conjunctivitis, urticaria, other documented allergies [via ATC code]) or prescription of an asthma or atopic dermatitis medication pre-index were used to determine co-morbidities. Patients were classified as publicly or privately insured. Prescriptions of concomitant allergic rhinoconjunctivitis (ARC) medication were based on record of a prescription one month pre-index or during the entire postindex period. The length of the post-index period varied between patients and ended when a patient no longer attended a physician in DA or on December 31, 2012.

\section{Results}

\section{Patient characteristics}

A total of 18,850 patients were prescribed AIT for grass pollen allergy during the study period. Patient characteristics are summarized in Table 1. Age of patients was consistent between AIT modalities, with a mean age of 12.1 years and 34.3 years in the paediatric and adult populations, respectively. In the paediatric population $63 \%$ of patients were male. $88 \%$ of patients were publicly insured. Five percent of patients had a record of a grass-related AIT prescription prior to index.

The most common co-morbidities observed in AIT patients at index were asthma (42\%), conjunctivitis (22\%), atopic dermatitis (20\%), and sinusitis (16\%).

Table 1 Patient characteristics at allergy immunotherapy initiation

\begin{tabular}{|c|c|c|c|}
\hline Characteristic & All patients $(n=18,850)$ & $<18$ years of age $(n=6425)$ & $\geq 18$ years of age $(n=12,425)$ \\
\hline$\overline{\mathrm{Age}^{a}}$ & $26.7 \pm 14.35(24.0)$ & $12.1 \pm 3.24(12.0)$ & $34.3 \pm 11.78(33.0)$ \\
\hline SLIT-tablets & $28.5 \pm 14.76(27.0)$ & $12.0 \pm 3.31(12.0)$ & $35.2 \pm 12.15(34.0)$ \\
\hline SLIT-drop & $26.5 \pm 15.44(25.0)$ & $11.1 \pm 3.41(11.0)$ & $35.9 \pm 11.97(35.0)$ \\
\hline SCIT & $26.2 \pm 14.09(24.0)$ & $12.2 \pm 3.20(12.0)$ & $33.9 \pm 11.61(32.0)$ \\
\hline Gender, male & $53 \%$ & $63 \%$ & $48 \%$ \\
\hline SLIT-tablets & $51 \%$ & $61 \%$ & $48 \%$ \\
\hline SLIT-drop & $53 \%$ & $65 \%$ & $46 \%$ \\
\hline SCIT & $54 \%$ & $64 \%$ & $48 \%$ \\
\hline \multicolumn{4}{|l|}{ Health insurance } \\
\hline State (public) & $16,502(88 \%)$ & $5729(89 \%)$ & 10,773 (87 \%) \\
\hline Private & $2348(12 \%)$ & $696(11 \%)$ & 1652 (13\%) \\
\hline Prior AIT usage at index & 957 (5 \%) & $351(5 \%)$ & $606(5 \%)$ \\
\hline SLIT-tablets & $31(1 \%)$ & $4(<1 \%)$ & $27(1 \%)$ \\
\hline SLIT-drop & $35(4 \%)$ & $11(3 \%)$ & $24(4 \%)$ \\
\hline SCIT & 891 (7 \%) & $336(7 \%)$ & $555(6 \%)$ \\
\hline \multicolumn{4}{|l|}{ Co-morbidities ${ }^{b}$} \\
\hline Asthma & 7904 (42 \%) & 2967 (46 \%) & $4937(40 \%)$ \\
\hline Conjunctivitis & $4215(22 \%)$ & 1709 (27 \%) & 2506 (20 \%) \\
\hline Atopic Dermatitis & $3770(20 \%)$ & $1634(25 \%)$ & $2136(17 \%)$ \\
\hline Sinusitis & $2954(16 \%)$ & 859 (13\%) & 2095 (17 \%) \\
\hline Concomitant ARC usage ${ }^{c}$ & 13,403 (71 \%) & 5053 (79 \%) & 8350 (67 \%) \\
\hline
\end{tabular}

a Mean \pm SD (median)

${ }^{\mathrm{b}}$ Co-morbidities not mutually exclusive

${ }^{\mathrm{c}}$ At index or post-index 
Co-morbidities were not mutually exclusive. Co-morbidities of other allergies and urticaria occurred in less than $15 \%$ of patients. The percentage of patients with a comorbidity was largely consistent across AIT modalities (77 \% SLIT-tablet, 81 \% SLIT drop, 79 \% SCIT). The majority (71\%) of patients had a record of concomitant ARC medication.

\section{Prescribing trends by AIT modality}

SCIT was the primary modality prescribed throughout the study period; however, there was a statistically significant increase in the proportion of patients treated with SLIT-tablets from $8 \%$ in $2006 / 2007$ to $29 \%$ in $2011 / 2012(p<0.001$, McNemar's test) (Fig. 1). The increase in SLIT-tablets between each prescribing year was also statistically significant $(p<0.001$, Chi-squared test for trend). Following SLIT-tablet approval in the paediatric population in 2008, its share of prescribing increased from $9 \%$ in 2008/09 to $26 \%$ in 2011/12. The proportion of patients prescribed SLIT-drops was marginal at 3-5\% of total prescribing each year; given this low usage, the results and discussion will focus on SCIT and SLIT-tablet modalities. Note: the sample of physicians increased throughout the study period (see "Characteristics of AIT prescribers" below), therefore the conclusion cannot be drawn that the number of patients treated with AIT increased.

\section{Prescribing trends by allergy season}

Initiation of SLIT-tablet and SCIT within each prescribing year generally had two peaks: autumn and the JanuaryApril period (Fig. 2). GRAZAX ${ }^{\circ}$ had a clear annual peak in September-November, while Oralair ${ }^{\circ}$ had a peak in initiations from January to March of each prescribing year; however, a second peak indicated a proportion of patients
(27\% on average) initiated Oralair ${ }^{\circ}$ from September to November. SCIT prescriptions exhibited a biphasic trend, with initial prescriptions peaking from September to October and in January. In the analysis of total prescriptions (not shown graphically) initial and total SCIT and Oralair prescriptions tracked each other closely. Total GRAZAX $^{\oplus}$ prescriptions oscillated throughout the year, indicative of its indication for year-round treatment. Less than $10 \%$ of all patients initiated treatment in-season (May-July).

\section{Characteristics of AIT prescribers}

AIT was prescribed by GPs, dermatologists, ENTspecialists, pediatricians and pneumologists (Fig. 3). The DA panel increased over the study period, with the number of physicians prescribing AIT increasing from 239 (2005) to 648 (2012). ENT-specialists and dermatologists together accounted for $67 \%$ of SCIT prescribing, while ENT-specialists accounted for $46 \%$ of SLIT-tablet prescribing.

Over the study period 756 unique physicians prescribed AIT to adult patients. These physicians treated a mean (SD) of 16.4 (30.5) adult AIT patients each. ENTspecialists were the largest prescribers of SLIT-tablets to adults (48\% of SLIT-tablet prescriptions). SCIT was predominantly prescribed by dermatologists and ENTspecialists, representing $42 \%$ and $37 \%$ of SCIT prescriptions, respectively. In 2012 ENT-specialists remained the largest prescribers of SLIT-tablets (50\% of prescriptions), while ENT-specialists and dermatologists together accounted for $79 \%$ of SCIT prescriptions.

A total of 617 unique physicians prescribed AIT to paediatric patients over the study period. These physicians treated a mean (SD) of 10.4 (16.3) paediatric AIT patients each. The largest prescribers of SLIT-tablet were

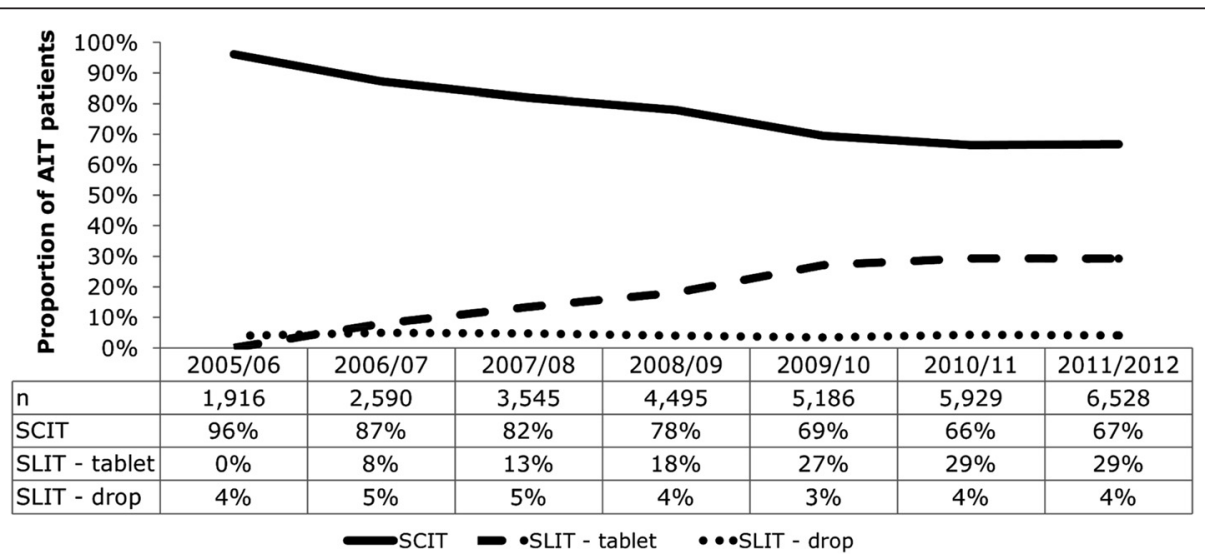

Fig. 1 First allergy immunotherapy prescription in each prescribing year, by modality. SCIT was the primary modality prescribed throughout the study period; however, there was a statistically significant increase in the proportion of patients treated with SLIT-tablets from $8 \%$ in 2006/2007 to $29 \%$ in 2011/2012 ( $p<0.001$, McNemar's test). The proportion of patients prescribed SLIT-drops was marginal at 3-5 \% of total prescribing each year 


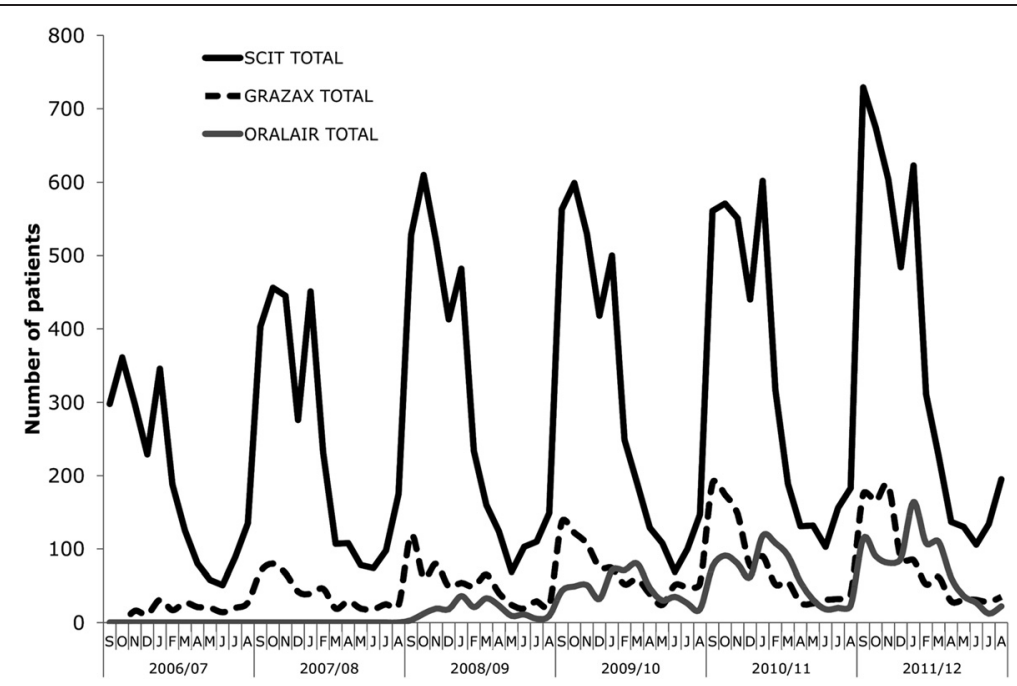

Fig. 2 Initiation of allergy immunotherapy, by prescribing season. Initiation of SLIT-tablet and SCIT within the prescribing year generally had two peaks: autumn and the January-April period. GRAZAX ${ }^{\circledR}$ had a clear annual peak in September-November, while Oralair ${ }^{\circledast}$ had a peak in initiations from January to March of each prescribing year; however, a second peak indicated a proportion of patients ( $27 \%$ on average) initiated Oralair ${ }^{\circledR}$ from September to November. SCIT prescriptions exhibited a biphasic trend, with initial prescriptions peaking from September to October and in January

ENT-specialists (43\% of SLIT-tablet prescriptions) while the largest prescribers of SCIT were paediatricians (39 \%). In 2012 SLIT-tablets were prescribed largely by ENT-specialists (50\%), while paediatricians accounted for $43 \%$ of SCIT prescriptions.

\section{Discussion}

\section{Main findings}

This retrospective cohort study of patients treated with AIT for grass pollen-induced AR in Germany uniquely

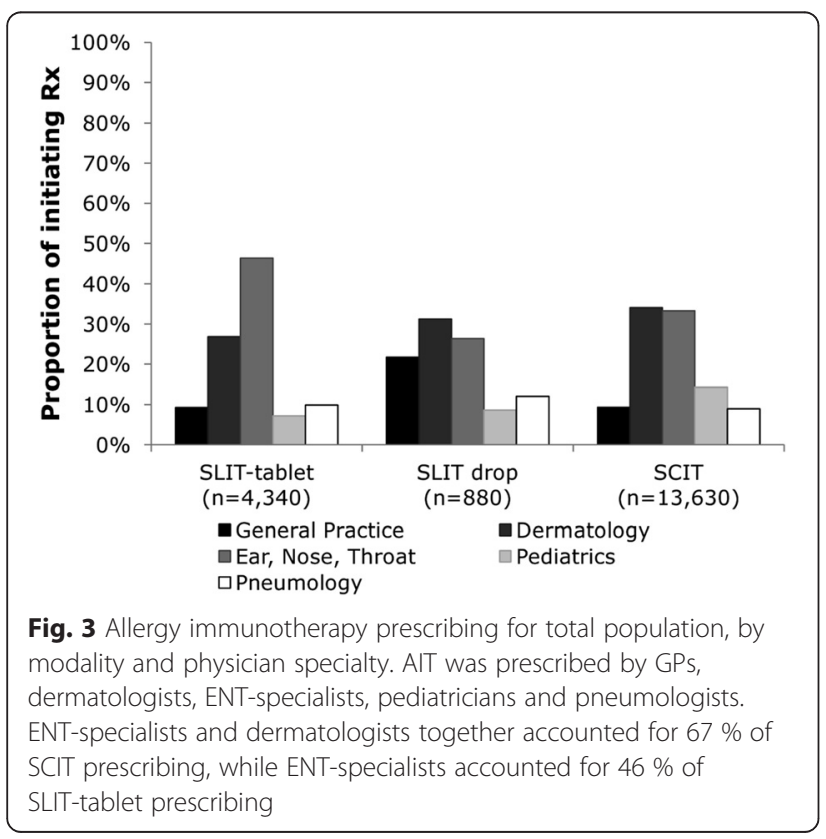

examined timing of initiation of treatment and prescribing trends over time. SCIT was the most commonly prescribed AIT modality, although the proportion of patients receiving SLIT-tablet increased markedly following launch in 2006. SLIT-drops had low utilization with a share of $3-5 \%$ of the total annual AIT prescribing across the study period. Initiation of SLIT-tablets GRAZAX ${ }^{\oplus}$ and Oralair ${ }^{\oplus}$ generally occurred in the autumn and JanuaryApril period, respectively, with a large proportion of Oralair ${ }^{\circledR}$ patients also receiving a prescription in autumn. SCIT had a biphasic trend, with prescriptions peaking in autumn and January. ENT-specialists and dermatologists were the largest AIT prescribers overall. The most common co-morbidities observed in patients at index were asthma, conjunctivitis, atopic dermatitis, and sinusitis.

\section{Our findings in context}

This is the first study of AIT prescribing trends in clinical practice that examines all available treatment modalities. Other studies have estimated the use of AIT among AR patients or focused on a specific route of AIT. Cross-sectional survey data and medical record review of seasonal AR patients in a small German sample indicated $35 \%$ of children/adolescents and $65 \%$ of adults received AIT [23], while claims data from 2007 to 2010 in Germany found $7 \%$ of patients with AR received AIT [24]. A previous publication reported that approximately $25 \%$ of AIT patients in Germany were treated with SLIT-tablets or SLIT-drops [25], whereas our study found that $33 \%$ of patients received SLIT-tablets or 
SLIT-drops (2011/2012 prescribing year). Biermann et al. [24] reported proportions of AR patients with co-morbid asthma ranging from $16.0 \%$ to $17.4 \%$ from 2007 to 2010 while asthma patients with co-morbid AR ranged from 47.3 to $48.1 \%$. The results of our study yielded estimates of co-morbid asthma of $40 \%$ in adult patients and $46 \%$ in paediatric patients prior to AIT initiation.

\section{Clinical relevance}

AIT is intended to induce immunologic tolerance hence pre-seasonal initiation for grass pollen allergies is ideal to minimize symptoms experienced during the season. The decision of when to initiate AIT should therefore be driven primarily by knowledge of timing of the grass pollen season. There was evidence that a large proportion of SLIT-tablet patients initiated treatment in the February-April time period, rather than four months in advance of the typical May-onset season as ideally recommended for optimal benefit. Furthermore, there was evidence that Oralair ${ }^{\oplus}$, a product recommended for use only until the end of the season, is initiated in the September-November period by an average of $27 \%$ of patients. While these patterns may reflect clinical experience surrounding the ability of patients to achieve adequate benefit with a shorter lead time (e.g., 12 weeks prior) or full treatment benefit with a greater lead time (e.g., prior autumn season), these differences likely impact the effectiveness and estimated cost of treatment.

The results also highlight insights on age of patients receiving AIT and type of physicians prescribing each modality. Given that AIT has been found to be welltolerated and effective in paediatric patients [16], it is interesting that only one-third of patients in the study sample were $<18$ years of age. This may indicate a need for education on proven benefit of AIT in paediatric patients, particularly given evidence that initiation of AIT in childhood may have additional benefits of preventing the onset of new sensitizations and asthma [2-10].

In terms of prescribing physician trends it is likely that physician preferences impact choice of modality. In the paediatric population paediatricians were the largest prescribers of SCIT, perhaps indicating a preference for this more established modality; whereas in both adult and paediatric patients ENT-specialists were the largest SLITtablet prescribers, possibly as it is more convenient to administer. In markets where allergy specialists administer AIT, the introduction of tablets may present greater complexity due to the need to consider multiple treatment options and patient characteristics suited to a particular modality. For other specialities, the oral SLIT-tablet may be viewed as a simpler approach to administering AIT. Furthermore, there may be economic incentives for SCIT due to the need for visits for injection. While country-bycountry variation in reimbursement, available modalities, and specialty types may impact prescribing trends, our findings can still be of interest to other markets, particularly as different physician specialties determine how to integrate new treatment modalities into their practice.

\section{Strengths \& limitations}

The primary strength of this study was the longitudinal examination of AIT prescriptions written for management of grass pollen-induced AR patients in clinical practice for a large sample of patients. The study also spanned the period pre- and post-SLIT-tablet launch, providing insight on the impact of this new modality on prescribing dynamics. Given the lack of other publications of real-world AIT usage, these findings contribute to the body of evidence of AIT practice.

Limitations of this study were the inability to link patients between physicians, meaning a patient's record included only the treatment provided by a single physician, and the reliance on physicians to accurately record information within the electronic medical records. Results may also have been impacted by the end of the German special allergy budget which changed reimbursement for administration of AIT during the study period.

\section{Conclusions}

As the first analysis of AIT prescribing trends in grass pollen-induced AR patients, this study highlighted the differences in onset of treatment initiation for different modalities and trends in prescribing by physician specialty. SCIT remained the dominant AIT modality in Germany, despite strong uptake of SLIT-tablets. Initiation of SCIT and Oralair generally peaked in two seasons while GRAZAX ${ }^{\circ}$ prescriptions peaked in autumn. ENT-specialists were the largest prescribers of SLITtablets, while paediatricians, dermatologists, and ENTspecialists were the largest SCIT prescribers. The study spanned a period where the treatment paradigm adapted to the introduction of the SLIT-tablet modality, with results showing predominance of particular modalities within certain physician specialties likely based on different treatment goals or needs. A possible reduction in the onset of future sensitizations and asthma through increased initiation of AIT in paediatric patients is an additional benefit that warrants further consideration. While the results are most relevant to clinical practice in Germany, they may reflect patterns in other countries with similar treatment paradigms.

\footnotetext{
Abbreviations

AIT: Allergy immunotherapy; AR: Allergic rhinitis; ARC: Allergic

rhinoconjunctivitis; ATC: Anatomical Therapeutic Chemical; DA: Disease

Analyzer; ENT: Ear, Nose and Throat; GP: General Practitioner;

SCIT: Subcutaneous immunotherapy; SLIT: Sublingual immunotherapy.
} 


\section{Competing interests}

FAR is employed by Merck \& Co. JNA is employed by ALK Abello. UW receives honoraria as a consultant for Merck \& Co, ALK-Abello, Allergopharma, Danone, Novartis, and Stallergenes. DD, AM, CR are employed by IMS Health. CH was employed by IMS Health at the time of the study. IMS Health was commissioned to conduct the study on behalf of Merck \& Co, and has ongoing consulting and research relationships with Merck \& Co.

\section{Authors' contributions}

AM co-wrote the study protocol, contributed to the interpretation of the results, and led manuscript development. UW contributed to the study protocol, the interpretation of the results, and the manuscript. DD contributed to the development of the study protocol, the interpretation of the results, and reviewed the manuscript. CR contributed to the development of the study protocol, extracted the patient-level data, conducted the analyses, contributed to the interpretation of the results, and reviewed the manuscript. $\mathrm{CH}$ co-wrote the study protocol, contributed to the interpretation of the results, and reviewed the manuscript. JNA contributed to the development of the study protocol, the interpretation of the results, and the manuscript. FAR contributed to the development of the study protocol, the interpretation of the results, and the manuscript. All authors read and approved the final manuscript.

\section{Acknowledgments}

This work was supported by Merck \& Co.

\section{Author details}

Real-World Evidence Solutions and Health Economics \& Outcomes Research, IMS Health, 210 Pentonville Road, London N1 9JY, UK. ²Department for Pediatric Pneumology and Immunology, Charité Medical University, Berlin, Germany. ${ }^{3}$ Worked at IMS Health at time of study, Real-World Evidence Solutions and Health Economics \& Outcomes Research, London, UK. ${ }^{4} \mathrm{Global}$ Market Access, ALK-Abelló A/S, Hørsholm, Denmark. ${ }^{5}$ Global Health Outcomes, Merck \& Co., West Point, PA 19486, USA

Received: 24 February 2015 Accepted: 20 May 2015

\section{Published online: 10 June 2015}

\section{References}

1. Bauchau $V$, Durham S. Prevalence and rate of diagnosis of allergic rhinitis in Europe. Eur Respir J. 2004;24:758-64.

2. Wilson $D$, Lime $M$, Durham SR. Sublingual immunotherapy for allergic rhinitis: systematic review and meta-analysis. Allergy. 2005;60:4-12.

3. Purello-D'Ambrosio F, Gangemi S, Merendino RA, Isola S, Puccinelli P, Parmiani $\mathrm{S}$, et al. Prevention of new sensitizations in monosensitized subjects submitted to specific immunotherapy or not: a retrospective study. Clin Experimental Allergy. 2001;31:1295-302.

4. Novembre E, Galli E, Landi F, Caffarelli C, Pifferi M, De Marco E, et al. Coseasonal sublingual immunotherapy reduces the development of asthma in children with allergic rhinoconjunctivitis. J Allergy Clin Immunol. 2004;114:851-7.

5. Möller C, Dreborg S, Ferdousi HA, Halken S, Høst A, Jacobsen L, et al. Pollen immunotherapy reduces the development of asthma in children with seasonal rhinoconjunctivitis (the PAT-study). J Allergy Clin Immu. 2002;109:251-6.

6. Marogna M, Tomassetti D, Bernasconi A, Colombo F, Massolo A, Di Rienzo Businco A, et al. Preventive effects of sublingual immunotherapy in childhood: an open randomized controlled study. Ann Allergy Asthma Immunol. 2008;101:206-11.

7. Jacobsen L, Niggemann B, Dreborg S, Ferdousi HA, Halken S, Høst A, et al. Specific immunotherapy has long-term preventive effect of seasonal and perennial asthma: 10-year follow-up on the PAT study. Allergy. 2007;62:943-8.

8. Jacobsen L. Preventive aspects of immunotherapy: prevention for children at risk of developing asthma. Ann Allergy Asthma Immunol. 2001;87:43-6.

9. Eng PA, Borer-Reinhold M, Heijnen IAFM, Gnehm HPE. Twelve-year follow-up after discontinuation of preseasonal grass pollen immunotherapy in childhood. Allergy. 2006;61:198-201.

10. Eng PA, Reinhold M, Gnehm HP. Long-term efficacy of preseasonal grass pollen immunotherapy in children. Allergy. 2002;57:306-12.

11. Didier A, Malling HJ, Worm M, Horak F, Jäger S, André C, et al. Optimal dose, efficacy, and safety of once-daily sublingual immunotherapy with a 5-grass pollen tablet for seasonal allergic rhinitis. J Allergy Clin Immu. 2007;120:1338-45.
12. Dahl R, Kapp A, Colombo G, de Monchy JGR, Rak S, Emminger W, et al. Efficacy and safety of sublingual immunotherapy with grass allergen tablets for seasonal allergic rhinoconjunctivitis. J Allergy Clin Immu. 2006;118:434-40.

13. Abramson MJ, Puy RM, Weiner JM. Injection allergen immunotherapy for asthma. Cochrane Database Syst Rev. 2010;8:CD001186.

14. Summary of Product Characteristics: Oralair 100 IR \& 300 IR sublingual tablets. http://www.stallergenes.com/fileadmin/images/corporate/ Emmanuelle/Oralair_SmPC.pdf (2013). Accessed 25 Nov 2014.

15. Summary of Product Characteristics: GRAZAX 75,000 SQ-T oral lyophilisate. http://www.medicines.orguk/emc/medicine/19565 (2014). Accessed 25 Nov 2014.

16. Cox L, Nelson H, Lockey R, Calabria C, Chacko T, Finegold I, et al. Allergen immunotherapy: a practice parameter third update. J Allergy Clin Immu. 2011;127:S1-55.

17. Canonica GW, Cox L, Pawankar R, Baena-Cagnani CE, Blaiss M, Bonini S, et al. Sublingual immunotherapy: World Allergy Organization position paper 2013 update. World Allergy Organ J. 2014;7:6.

18. Nelson HS. Subcutaneous immunotherapy versus sublingual immunotherapy: which is more effective? J Allergy Clin Immunol Pract. 2014;2:144-9.

19. Dretzke J, Meadows A, Novielli N, Huissoon A, Fry-Smith A, Meads C. Subcutaneous and sublingual immunotherapy for seasonal allergic rhinitis: a systematic review and indirect comparison. J Allergy Clin Immunol. 2013;131:1361-6.

20. Hamilton JG. Needle phobia: a neglected diagnosis. J Fam Pract. 1995:41:169-75

21. Cox L, Calderon MA. Subcutaneous specific immunotherapy for seasonal allergic rhinitis: a review of treatment practices in the US and Europe. Curr Med Res Opin. 2010;26:2723-33.

22. Becher H, Kostev K, Schröder-Bernhardi D. Validity and representativeness of the "Disease Analyzer" patient database for use in pharmacoepidemiological and pharmacoeconomic studies. Int J Clin Pharm Th. 2009;47:617-26

23. Schramm B, Ehlken B, Smala A, Quednau K, Berger K, Nowak D. Cost of illness of atopic asthma and seasonal allergic rhinitis in Germany: 1-yr retrospective study. Eur Respir J. 2003;21:116-22.

24. Biermann J, Merk HF, Wehrmann W, Klimek L, Wasem J. Allergic disorders of the respiratory tract - findings from a large patient sample in the German statutory health insurance system. Allergo J. 2013:22:366-73.

25. Cox L, Jacobsen L. Comparison of allergen immunotherapy practice patterns in the United States and Europe. Ann Allergy Asthma Immunol. 2009;103:451-60

\section{Submit your next manuscript to BioMed Central and take full advantage of:}

- Convenient online submission

- Thorough peer review

- No space constraints or color figure charges

- Immediate publication on acceptance

- Inclusion in PubMed, CAS, Scopus and Google Scholar

- Research which is freely available for redistribution 